\title{
Observations of a pulse-driven cool polar jet by SDO/AIA
}

\author{
A. K. Srivastava ${ }^{1,2}$ and K. Murawski ${ }^{1}$ \\ ${ }^{1}$ Group of Astrophysics, UMCS, ul. Radziszewskiego 10, 20-031 Lublin, Poland \\ e-mail: kmur@kft.umcs.lublin.pl \\ 2 Aryabhatta Research Institute of Observational Sciences (ARIES), Manora Peak, Nainital-263 129, Uttarakhand, India \\ e-mail: aks@aries.res.in
}

Received 27 May 2011 / Accepted 18 August 2011

\section{ABSTRACT}

\begin{abstract}
Context. We observe a solar jet at north polar coronal hole (NPCH) using SDO AIA $304 \AA$ image data on 3 August 2010. The jet rises obliquely above the solar limb and then retraces its propagation path to fall back.

Aims. We numerically model this solar jet by implementing a realistic (VAL-C) model of solar temperature.

Methods. We solve two-dimensional ideal magnetohydrodynamic equations numerically to simulate the solar jet. We consider a localized velocity pulse that is essentially parallel to the background magnetic field lines and is initially launched at the top of the solar photosphere. The pulse steepens into a shock at higher altitudes, which triggers plasma perturbations that exhibit the observed features of the jet. The typical direction of the pulse also clearly exhibits the leading front of the moving jet.

Results. Our numerical simulations reveal that a large amplitude initial velocity pulse launched at the top of the solar photosphere in general produces the observed properties of the jet, e.g., upward and backward average velocities, height, width, life-time, and ballistic nature.

Conclusions. The close match between the jet observations and numerical simulations provides a first strong evidence that this jet is formed by a single velocity pulse. The strong velocity pulse is most likely generated by the low-atmospheric reconnection in the polar region, which triggers the jet. The downflowing material of the jet most likely is absorbed in the next upcoming velocity pulses from the lower solar atmosphere, and because of that we only see a single jet moving upward in the solar atmosphere.
\end{abstract}

Key words. Sun: corona - magnetohydrodynamics (MHD) - Sun: chromosphere

\section{Introduction}

Solar jets are the typical collimated and short-lived transient ejecta in the solar atmosphere that are significant in mass and energy transport at various spatio-temporal scales. Based on the spatial sizes, life-times, and transient nature, the jets are classified in the form of various phenomena, e.g., anemone jets (Shibata et al. 2007), EUV jets (Innes et al. 1997), spicules (Bohlin et al. 1975), X-ray jets (Shibata et al. 1992), and penumbral jets (Katsukawa et al. 2007). The solar surges are cool jets typically formed with a plasma that is usually visible in $\mathrm{H}_{\alpha}$ and other chromospheric lines, and they are supposed to be triggered by magnetic reconnection processes (Brooks et al. 2007). However, solar surges are mostly associated with the flaring regions and the places of transient activities at the Sun. Georgakilas et al. (1999) have also observed the polar surges that were cool surge-like jets over polar caps formed without any transient phenomenon. However, these jets were associated with macro-spicules, and because of their short-lived nature they could not attain the greater heights in the off-limb polar corona.

Other short spatio-temporal scale jets (e.g., dynamic fibrils, mottles, and spicules) are also significant low-atmospheric phenomena that are necessary to understand energy and mass transport in the solar atmosphere. Using high-resolution observations from the $1 \mathrm{~m}$ Swedish Solar Telescope and advanced numerical modeling, Hansteen et al. (2006) have shown that in the active regions these jets are a natural consequence of upward propagating slow-mode magnetoacoustic shocks generated by the leakage of $\mathrm{p}$-mode subphotospheric oscillations. The active region fibrils are also most likely formed by chromospheric shock waves that occur when convective flows and global oscillations leak into the chromosphere along the field lines of magnetic flux concentrations (De Pontieu et al. 2007a). De Pontieu et al. (2004) have also reported for the first time the quasi-periodic rise and fall of the spicule material caused by the leakage of p-mode oscillations from the subphotospheric regions. De Pontieu et al. (2007b) have pointed out that typical type-I spicules are found to be driven by shock waves that form when global oscillations and convective flows leak into the upper atmosphere along magnetic field lines on timescales of 3-7 min. These authors have also found the signature of type-II spicules, which are much more dynamically formed within $10 \mathrm{~s}$ and are very thin $(200 \mathrm{~km}$ wide $)$ with life-times of 10-150 s. These types of spicules seem to be rapidly heated to transition region temperatures and eject material through the chromosphere at speeds of $50-150 \mathrm{~km} \mathrm{~s}^{-1}$. The properties of type-II spicules suggest a formation process that is a consequence of magnetic reconnection, typically in the vicinity of magnetic flux concentrations in plage and network regions. Recently, Morton et al. (2011) have reported a unique signature of the relationship between multiple ejecta and the polar-jet formation using the SDO/AIA observations However, the relationship between the various types of jets are still not satisfactorily explored.

In various types of these jet phenomena, the coronal and polar coronal jets are classic transient phenomena that have been observed by Skylab and Yohkoh in soft X-rays (Shimojo \& Shibata 2000), followed by the Solar and Heliospheric Observatory (SoHO, Wang et al. 1998), the Transition Region and Coronal Explorer (TRACE, Alexander \& Fletcher 1999), and Hinode (Kamio et al. 2007; Filippov et al. 2009). The 


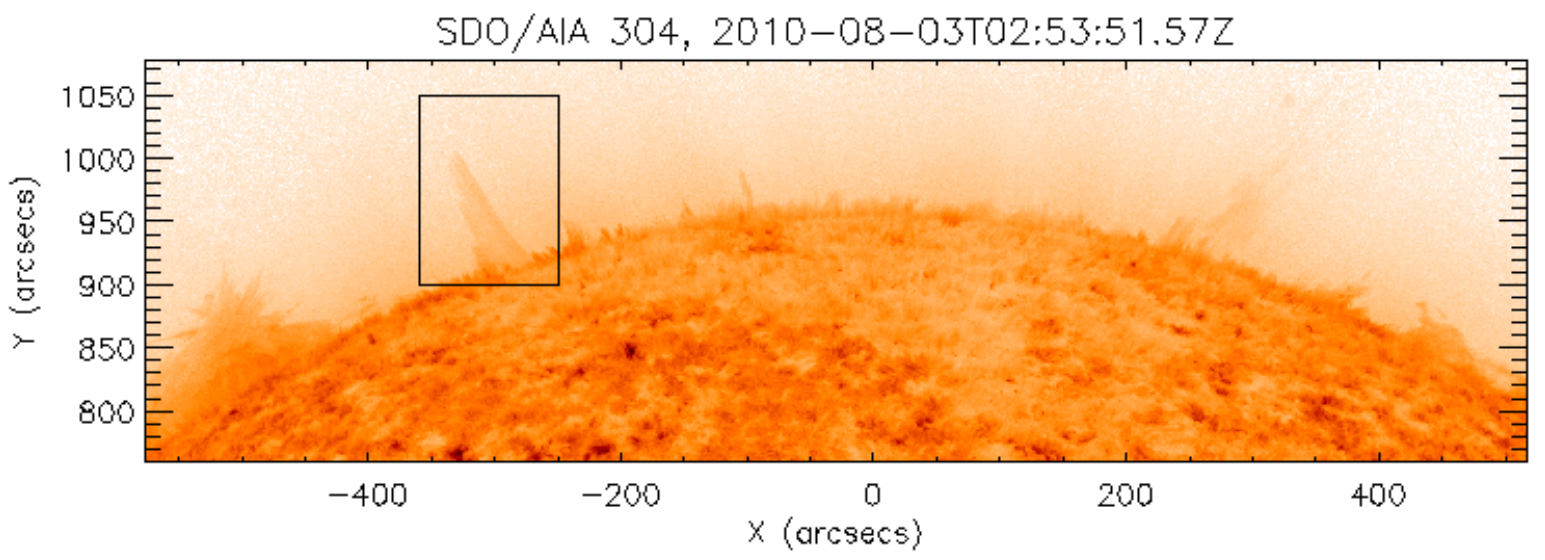

Fig. 1. Field-of-view (FOV) of the north polar region as observed by SDO/AIA $304 \AA$ on 03 August 2010 at 02:53:51 UT. The $\left(X_{\text {cen }}, Y_{\text {cen }}\right)$ was $\left(-29.618^{\prime \prime}, 918.631^{\prime \prime}\right)$, while the full FOV was $\left(1094^{\prime \prime}, 317^{\prime \prime}\right)$ wide with a resolution of $0.6^{\prime \prime}$ per pixel. The black box shows the triggering of a solar jet that exhibits an exceptional phenomenon of its most probable formation by a velocity pulse.

magnetic reconnection is found to be one of the most likely mechanisms for the generation of typical coronal jets (Shibata et al. 2007), while the reconnection-generated waves may also be present in the jets during their propagation (Yokoyama \& Shibata 1995, 1996). Pariat et al. (2009) have recently simulated the propagation of kink motion along the magnetic field lines around a rotating and closed magnetic field region. This dynamics is most likely associated with the kink instability in the jet-formation region, which may lead its upward motion in the model solar atmosphere. The transversal waves have also been recently detected in connection with the solar jet events at small spatial scales (e.g., He et al. 2009; Kamio et al. 2010, and references cited there). However, the evidence of Alfvén waves has also been detected in the large-scale polar X-ray jets (Cirtain et al. 2007). Therefore, the magnetic reconnection and associated waves may be the most likely drivers to energize this type of jet at various spatio-temporal scales. However, the exact mechanism is not sufficiently established in the context of the very complex motion of the various types of solar jets.

Recently, Murawski \& Zaqarashvili (2010) have reported the formation of solar spicules by a pulse launched within the chromosphere. The authors' numerical simulations show that the strong initial pulse may lead to the quasi-periodic rising of the chromospheric material into the lower corona in the form of spicules. The authors have explained the observed speed, width, and heights of type-I spicules, as well as observed multistructural and bi-directional flows with a quasi-periodic rise and fall at 3-5 min time scales caused by the consecutive shocks. The reconnection-generated velocity pulses may also drive the solar jets. However, such models along with their observational supports are not yet available for solar jets. Therefore, it may be worth to explore the launching of a solar jet through the formation of a pulse in the lower solar atmosphere.

In the present paper, we find a unique observational signature of a cool jet off the limb at the north-east boundary of a moderately evolved coronal hole in the northern polar region that shows dynamics driven by a velocity pulse. We numerically simulate a similar kind of jet triggered by a strong amplitude velocity pulse in the lower solar atmosphere, which generaly closely mimics the observed properties of the jet. In Sect. 2 we describe the observational data and analyses. We report the numerical model in Sect. 3 and results of numerical simulation in Sect. 4. We present the discussion and conclusions in the last section.

\section{Observations and results}

We use a time-series of the data of a solar jet at the north polar region as observed in the $304 \AA$ filter of Atmospheric Assembly Imager (AIA) onboard the Solar Dynamics Observatory (SDO) on 3 August 2010 during 02:41:14 UT-03:02:50 UT. The SDO/AIA has a typical resolution of $0.6^{\prime \prime}$ per pixel and the highest cadence of $12 \mathrm{~s}$, and it observes the full solar disk in three UV (1600 $,, 1700 \AA, 4500 \AA)$ and seven EUV (171 , $193 \AA, 211 \AA$, $94 \AA, 304 \AA$, $335 \AA$ 兄 $131 \AA$ ) wavelengths (Lemen et al. 2011). Therefore, it provides unprecedented observations of multi-temperature, high-resolution, and high-temporal plasma dynamics across the whole Sun. The field-of-view of the north polar region as observed by SDO/AIA $304 \AA$ on 03 August 2010 at 02:53:51 UT is shown in Fig. 1 . The $\left(X_{\text {cen }}, Y_{\text {cen }}\right)$ was $\left(-29.618^{\prime \prime}, 918.631^{\prime \prime}\right)$, while the full FOV was (1094", 317") wide with a resolution of $0.6^{\prime \prime}$ per pixel. The black box shows the triggering of a solar jet off the polar limb. The time series has been obtained by the SSW cutout service at LMSAL, USA, which is corrected for the flat-field and spikes. We ran the aia_prep subroutine of SSW IDL also for subsequent calibration and cleaning of the time-series data.

We observe a unique jet formation in the east-north limb of the polar coronal hole, which seems to be driven by a velocity pulse launched in the lower solar atmosphere. The jet has been launched slightly obliquely from the background open field lines of the polar coronal hole (cf., Fig. 2). The life-time of the jet was observed to be $\sim 21 \mathrm{~min}$, which is the typical life-time of the coronal jets that were previously observed in the Sun and cataloged by Nisticò et al. (2009). We observe a typical jet that reaches into the corona off the polar limb. However, the jet material was only visible in He II $304 \AA$ filter, which is sensitive to the comparatively cool temperature of $1 \times 10^{5} \mathrm{~K}$. The jet material could not have been evident in the typical TR/coronal filters (e.g., Fe IX $171 \AA$, $211 \AA$ filters) of SDO/AIA, which are sensitive to the plasma temperature $\geq 10^{6} \mathrm{~K}$. Figure 2 shows the SDO/AIA $171 \AA$ (left) and $211 \AA$ (right) coronal images overlaid by SDO/AIA $304 \AA$ intensity contours at a time of the maximum rise of the jet. Evidently the coronal filters do not exhibit much sensitivity to the jet plasma, which is only observed in the He II $304 \AA$ filter. This scenario persists during whole life-time of the jet. The unique jet is a very faint event even captured in $304 \AA$ because most of the strong emissions come from spicule borders at this wavelength as well. However, we cannot rule out some very weak amount of coronal emissions 

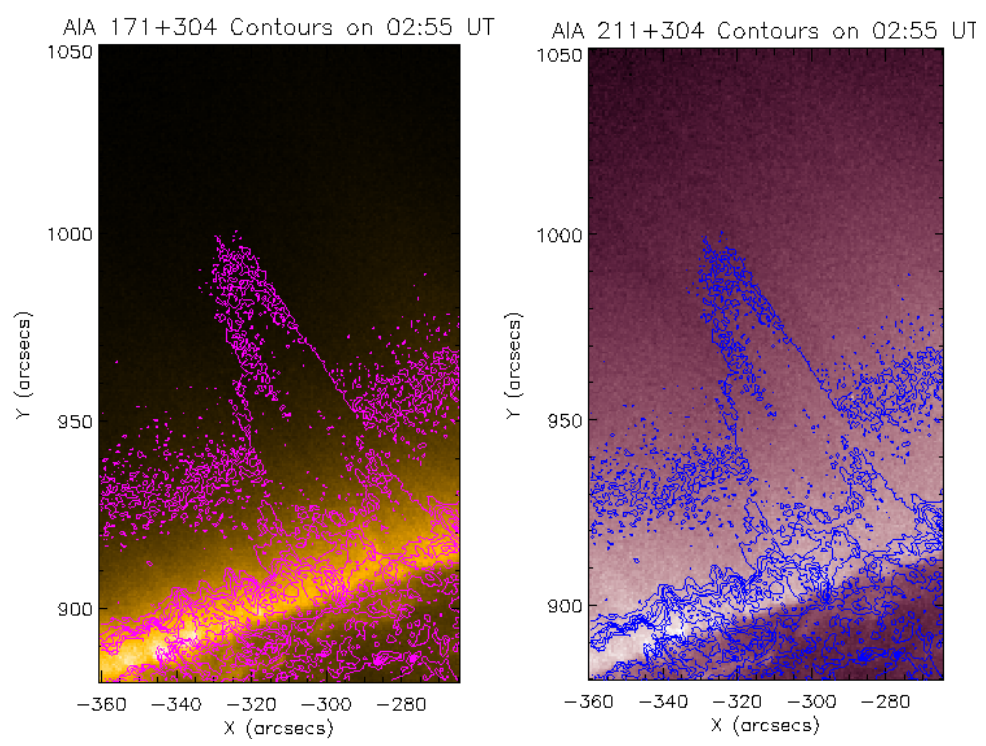

Fig. 2. SDO/AIA $171 \AA$ (left) and $211 \AA$ (right) coronal images overlaid by SDO/AIA $304 \AA$ intensity contours. Evidently the coronal filters do not reveal much sensitivity to the jet plasma, which is visible in the He II 304 A filter.

that could not be distinguished in the diffused background off the polar limb. Therefore, we consider this jet a cool jet because its plasma mostly emits the radiations captured by the AIA filters that are sensitive to cooler plasma emissions, e.g., $304 \AA$. Possibly there are also emissions in other SDO/AIA filters that are sensitive to the emissions from comparatively cooler plasma. This means that the jet material was typically formed by comparatively cooler plasma compared to the background coronal plasma, and it moves in form of a plasma column that does not exchange any heat with the ambient coronal plasma. This was the unique property of the observed cool jet off-limb in the polar coronal hole. Our observed cool ejecta reaches a higher height $(\sim 72 \mathrm{Mm})$ with a life-time of $\sim 21 \mathrm{~min}$ and larger width of $\sim 20 \mathrm{Mm}$. We therefore consider it a cool polar jet and not an evidence of any polar surge ejecta.

The jet starts moving above the solar limb on $\sim 02: 41$ UT and reaches a maximum height of $\sim 72 \mathrm{Mm}$ at 02:52 UT. Therefore, its average rising speed is $\sim 110 \mathrm{~km} \mathrm{~s}^{-1}$, which is the typical speed of the jet with which it rose in the corona at a maximum height of $\sim 72 \mathrm{Mm}$ in the first $\sim 648 \mathrm{~s}$ (Fig. 3). Although the width slightly varies at the base of the jet, its shape is approximately uniform during its rise. Its width at the base is measured as $\sim 20 \mathrm{Mm}$. The unique evidence this jet presented is its rise straight into the corona off-limb in the first 10-11 min of its life-time, and its subsequent fall back along the same path. Some unique observations of chromospheric jet with the Hinode/SOT Ca II H filter revealed fine structuring as well as transversal motion, which was associated with the helicity injection in the upper solar atmosphere (Liu et al. 2009). However, the spatial resolution of SDO/AIA $\left(0.6^{\prime \prime}\right)$ is indeed lower compared to SOT $\left(0.2^{\prime \prime}\right)$, and the cool jet is much fainter, which impedes a clear identification of this fine structure. Moreover, this jet (Liu et al. 2009) was associated with a small flare at the western limb, while in the present case we observe the cool jet in a polar coronal hole. Therefore, these two cases are entirely different, and we do not observe this type of oscillating threads or helicity injection.

The similar type of the dynamics in form of the parabolic trajectory has been reported for various types of spicules (type I and II), dynamic fibrils, mottles (Hansteen et al. 2006; De Pontieu et al. 2007a,b, 2011). These findings also support the assumption that the acceleration of these small-scale jets by the shock triggered in the chromosphere is caused by velocity pulses generated by the leakage of subphotospheric p-modes. However, these drivers need several $\mathrm{km} \mathrm{s}^{-1}$ velocity amplitude, 3-5 min temporal scales, and some concentrated field regions of a few hundred Gauss to push these jets 2-10 Mm above the solar limb. Recently, Murawski \& Zaqarashvili (2010) have also simulated the solar spicules by launching a velocity pulse above the solar photosphere. However, a strong velocity pulse will be needed in the present case to trigger jet material higher in the corona.

Our observed dynamics supports that this jet may also be formed by a velocity pulse that was triggered in the chromosphere and steepened in form of a shock in the transition region/corona. However, it may not be associated with the typical photospheric velocity pulses on the order of few $\mathrm{km} \mathrm{s}^{-1}$ associated with the leakage of $\mathrm{p}$-modes that launch the small-scale jets, as mentioned in several previous studies (Hansteen et al. 2006; De Pontieu et al. 2007a,b). In the present case, the steepened shock can be generated by a reconnection-generated strong velocity pulse. Its front may rarefy the plasma density behind it, and the jet plasma would be able to rise. After reaching into the corona along curved magnetic field lines, this jet falls back owing to gravity and the cool plasma retraces its path, as observed here. In the next section we discuss in detail the numerical simulation of the observed jet in the solar atmosphere triggerred by a strong velocity pulse.

\section{A numerical model}

Our model system is taken to be composed of a gravitationallystratified solar atmosphere that is described by the ideal twodimensional (2D) MHD equations:

$$
\begin{array}{r}
\frac{\partial \varrho}{\partial t}+\nabla \cdot(\varrho \boldsymbol{V})=0, \\
\varrho \frac{\partial \boldsymbol{V}}{\partial t}+\varrho(\boldsymbol{V} \cdot \nabla) \boldsymbol{V}=-\nabla p+\frac{1}{\mu}(\nabla \times \boldsymbol{B}) \times \boldsymbol{B}+\varrho \boldsymbol{g}, \\
\frac{\partial p}{\partial t}+\nabla \cdot(p \boldsymbol{V})=(1-\gamma) p \nabla \cdot \boldsymbol{V}, \\
\frac{\partial \boldsymbol{B}}{\partial t}=\nabla \times(\boldsymbol{V} \times \boldsymbol{B}), \quad \nabla \cdot \boldsymbol{B}=0 .
\end{array}
$$


A\&A 534, A62 (2011)
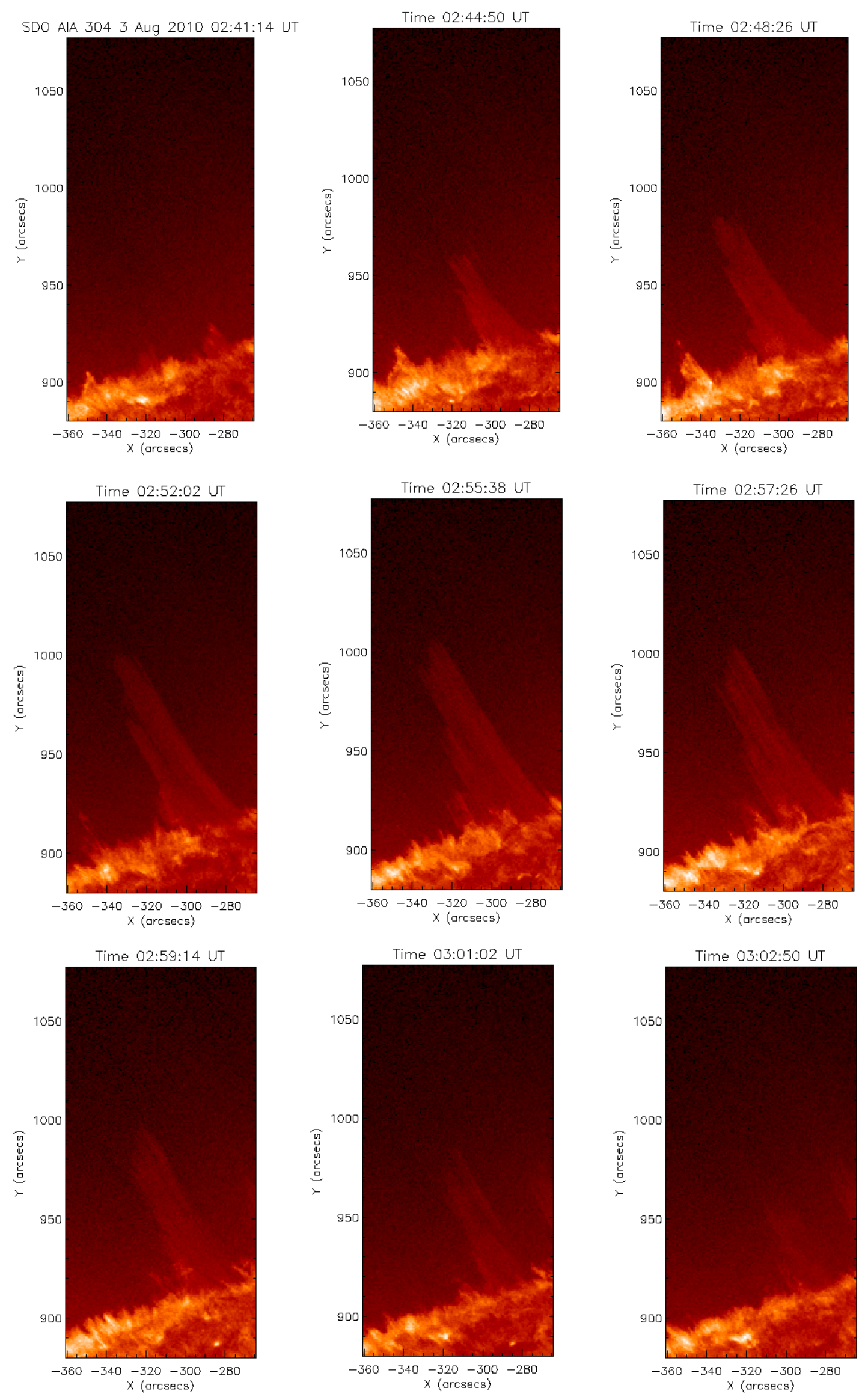

Fig. 3. SDO/AIA $304 \AA$ time sequence of a cool jet propagation above the solar limb in the northeast region of a polar coronal hole that clearly shows the upward motion of the plasma and its downward motion along the same path. 
Here $\varrho$ is mass density, $\boldsymbol{V}$ is the flow velocity, $\boldsymbol{B}$ is the magnetic field, $p=\frac{k_{\mathrm{B}}}{m} \varrho T$ is the gas pressure, $T$ is the temperature, $\gamma=5 / 3$ is the adiabatic index, $\boldsymbol{g}=(0,-g)$ is the gravitational acceleration of its value $g=274 \mathrm{~m} \mathrm{~s}^{-2}, m$ is the mean particle mass and $k_{\mathrm{B}}$ is Boltzmann's constant.

\subsection{Equilibrium configuration}

We assume that at its equilibrium the solar atmosphere is still $\left(\boldsymbol{V}_{\mathrm{e}}=0\right)$ with a force-free magnetic field,

$\left(\nabla \times \boldsymbol{B}_{\mathrm{e}}\right) \times \boldsymbol{B}_{\mathrm{e}}=0$,

such that it satisfies a current-free condition, $\nabla \times \boldsymbol{B}_{\mathrm{e}}=0$, and it is specified by the magnetic flux function, $A$, as

$\boldsymbol{B}_{\mathrm{e}}=\nabla \times(A \hat{z})$

Here the subscript e corresponds to equilibrium quantities. We set an arcade magnetic field by choosing

$A(x, y)=B_{0} \Lambda_{\mathrm{B}} \cos \left(x / \Lambda_{\mathrm{B}}\right) \exp \left[-\left(y-y_{\mathrm{r}}\right) / \Lambda_{\mathrm{B}}\right]$

Here, $B_{0}$ is the magnetic field at $y=y_{\mathrm{r}}$, and the magnetic scaleheight is

$\Lambda_{\mathrm{B}}=2 L / \pi$.

We set and hold fixed $L=100 \mathrm{Mm}$.

As a result of Eq. (5) the pressure gradient is balanced by the gravity force,

$-\nabla p_{\mathrm{e}}+\varrho_{\mathrm{e}} \boldsymbol{g}=0$.

With the ideal gas law and the $y$-component of Eq. (8), we arrive at

$p_{\mathrm{e}}(y)=p_{0} \exp \left[-\int_{y_{\mathrm{r}}}^{y} \frac{\mathrm{d} y^{\prime}}{\Lambda\left(y^{\prime}\right)}\right], \quad \varrho_{\mathrm{e}}(y)=\frac{p_{\mathrm{e}}(y)}{g \Lambda(y)}$,

where

$\Lambda(y)=k_{\mathrm{B}} T_{\mathrm{e}}(y) /(m g)$

is the pressure scale-height, and $p_{0}$ denotes the gas pressure at the reference level that we choose in the solar corona at $y_{\mathrm{r}}=$ $10 \mathrm{Mm}$.

We adopt an equilibrium temperature profile $T_{\mathrm{e}}(z)$ for the solar atmosphere that is close to the VAL-C atmospheric model of Vernazza et al. (1981). Then with Eq. (9) we obtain the corresponding gas pressure and mass density profiles.

\subsection{Perturbations}

We initially perturb the above equilibrium impulsively by a Gaussian pulse in the vertical component of velocity, $V_{y}$, viz.,

$V_{y}(x, y, t=0)=A_{\mathrm{v}} \exp \left[-\frac{\left(x-x_{0}\right)^{2}}{w_{x}^{2}}-\frac{\left(y-y_{0}\right)^{2}}{w_{y}^{2}}\right]$.

Here $A_{\mathrm{v}}$ is the amplitude of the pulse, $\left(x_{0}, y_{0}\right)$ is its initial position and $w_{x}, w_{y}$ denotes its width along the $x$ - and $y$-directions, respectively. We set and hold fixed $A_{\mathrm{v}}=160 \mathrm{~km} \mathrm{~s}^{-1}, x_{0}=$ $-17.5 \mathrm{Mm}, y_{0}=1.75 \mathrm{Mm}, w_{x}=10 \mathrm{Mm}$, and $w_{y}=0.1 \mathrm{Mm}$.

\section{Results of the numerical simulations}

Equations (1)-(4) are solved numerically using the code FLASH (Lee \& Deane 2009). This code implements a second-order unsplit Godunov solver with various slope limiters and Riemann solvers, as well as adaptive mesh refinement (AMR). We set the simulation box of $(-125,15) \mathrm{Mm} \times(0,110) \mathrm{Mm}$ along the $x$ and $y$-directions and impose fixed in time all plasma quantities at all four boundaries of the simulation region. In all our studies we use an AMR grid with a minimum (maximum) level of refinement set to 4 (8). The refinement strategy is based on controlling numerical errors in mass density, which results in an excellent resolution of steep spatial profiles and greatly reduces numerical diffusion at these locations.

Figure 4 displays the spatial profiles of the plasma temperature (color maps) and velocity (arrows) resulting from the initial velocity pulse of Eq. (11), which splits into counter-propagating parts. The part that propagates downward is reflected from the dense plasma layers at the photospheric region. This reflected part lags behind the originally upward propagating signal, which becomes a shock. Because the plasma is initially pushed upward, the under-pressure results in the region below the initial pulse. This under-pressure sucks up comparatively cold chromospheric plasma, which lags behind the shock front at higher temperature. As a result, the pressure gradient force works against gravity and forces the chromospheric material to penetrate into the solar corona in form of a rarefaction wave. At $t=25 \mathrm{~s}$ this shock reaches the altitude of $y \simeq 10 \mathrm{Mm}$ and the rarefaction wave is located $1 \mathrm{Mm}$ below the slow shock. The next snapshot (top middle panel) is drawn for $t=200 \mathrm{~s}$. At this time the slow shock reached the altitude of $y \simeq 63 \mathrm{Mm}$, while the chromospheric plasma is located below at $y \simeq 40 \mathrm{Mm}$. At the next moment, $t=500 \mathrm{~s}$ (top right panel) the shock moves up and the chromospheric plasma blob shows its developed phase, reaching the level of $y=64 \mathrm{Mm}$, which matches the observational data of Fig. 3 well (top right panel). The cool jet slows down while propagating upward. At $t=800 \mathrm{~s}$ it arrives at the level of $y \simeq 70 \mathrm{Mm}$ (Fig. 3, middle-right panel) and subsequently subsides as the plasma begin to flow downward, because it is attracted by gravity (bottom panels). At the subsiding stage the fine structures develop as a result of interaction between the downfalling plasma and the rising secondary pulse. Similar features were visible in the numerical simulation of spicules (Murawski \& Zaqarashvili 2010).

\section{Discussions and conclusion}

Our numerical simulations show a general scenario of rising and subsequently down-falling cool plasma jet with a number of observational features such as jet height and width, ballistic motion, approximate rising time-scale and average rising velocity that are close to the observational data. However, some parts of the match between the numerical and observational data are approximate and only qualitative, e.g., the rising time-scale in the simulation is $\sim 800 \mathrm{~s}$, while in the real observations it is $\sim 720 \mathrm{~s}$. This mismatch may obviously result from some of the simplified profiles of the model parameters in our numerical model, e.g., the magnetic field configuration at a place at which the initial pulse was launched, and so on. Moreover, the real jet was excited in more complex plasma and magnetic field conditions at the polar coronal hole of the Sun. We may not be entirely accurate in our modeling of the way in which the jet was excited in the real Sun. Indeed, the exciter could work for some time, it could be located at a different place and it could have a different size, speed and distribution. Because these data are 


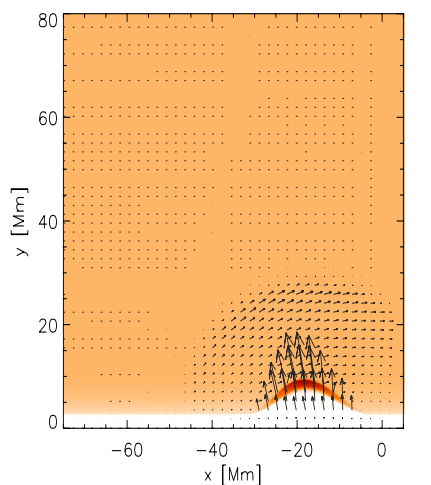

$x[\mathrm{Mm}]$

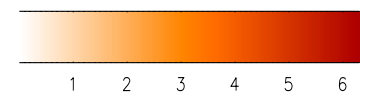

$\uparrow 0.15$
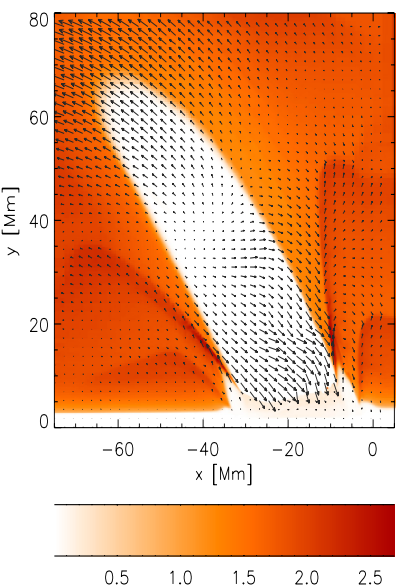

$\longleftrightarrow 0.15$

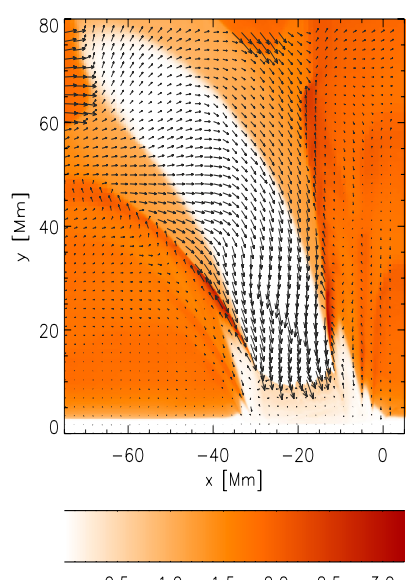

$\uparrow \quad 0.15$
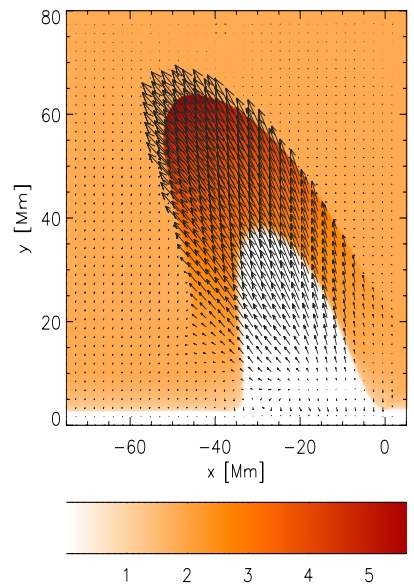

^ 0.15
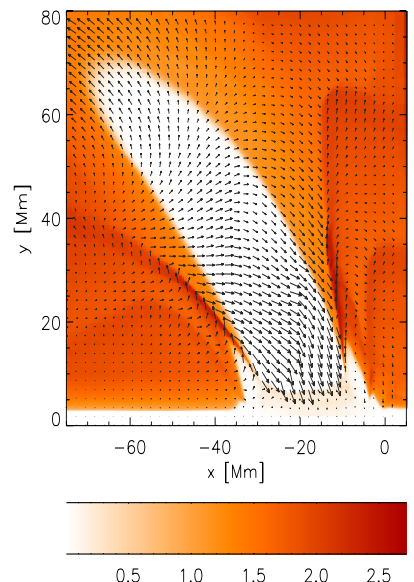

$\uparrow 0.15$
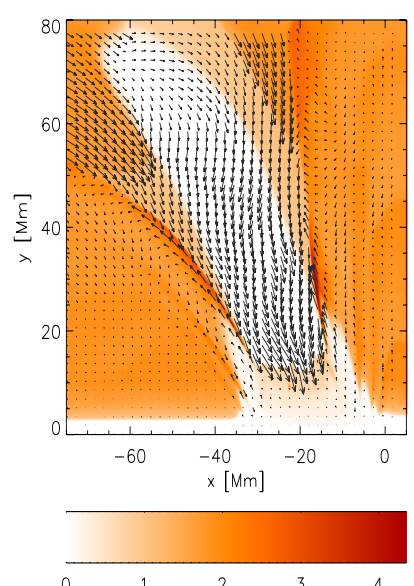

0.15
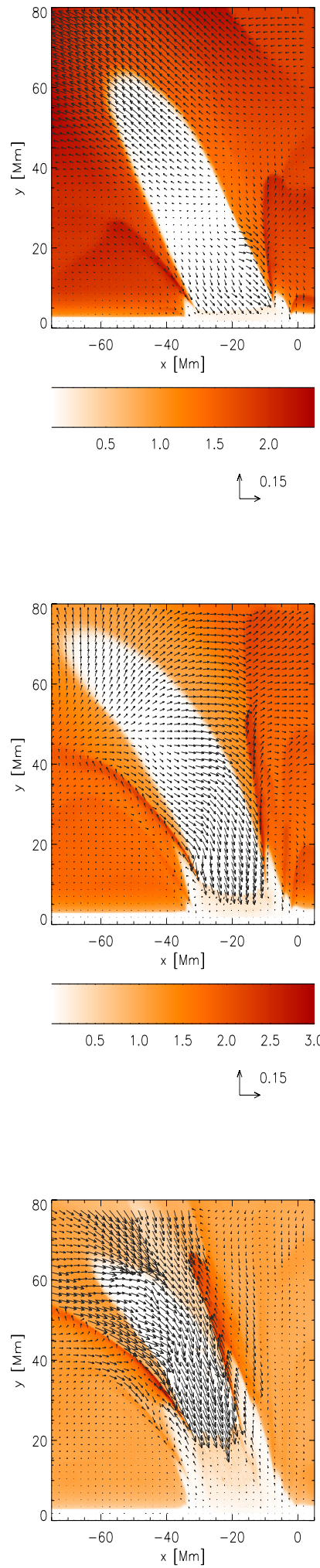

^ 0.15
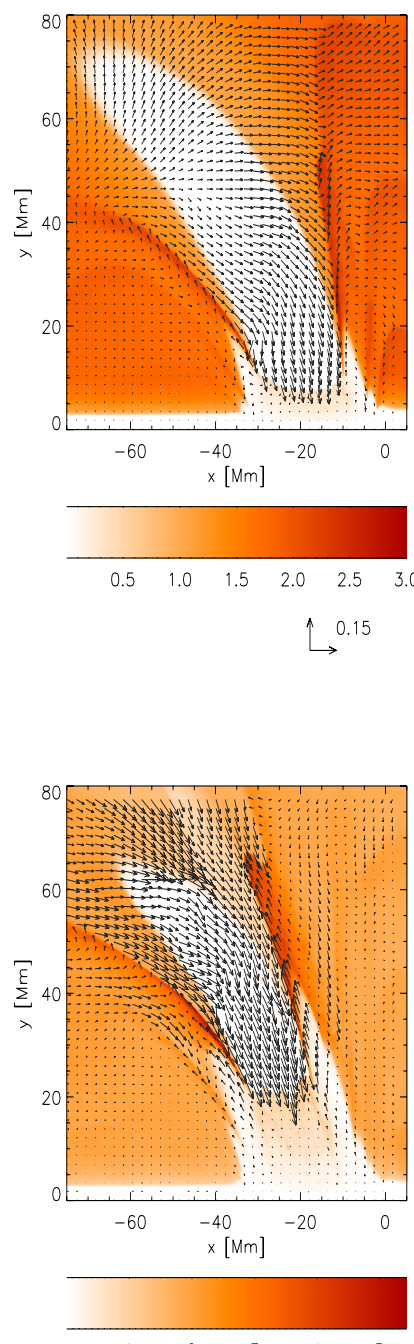

0.15

Fig. 4. Temperature (color maps) and velocity (arrows) profiles at $t=25 \mathrm{~s}, t=200 \mathrm{~s}, t=500 \mathrm{~s}, t=600 \mathrm{~s}, t=700 \mathrm{~s}, t=800 \mathrm{~s}, t=900 \mathrm{~s}$, $t=1000 \mathrm{~s}$, and $t=1200 \mathrm{~s}$ (from top-left to bottom-right). Temperature is drawn in units of $1 \mathrm{MK}$. The arrow below each panel represents the length of the velocity vector, expressed in units of $150 \mathrm{~km} \mathrm{~s}^{-1}$. 
not provided by the observations, we decided to trigger the jet by a localized pulse in a plasma velocity and launch this jet below the transition region. In this way we managed to excite a jet that mimics on average the properties of the cool plasma jet. The small mismatches in the exact height and length of the jet as well as its descending time can be reduced by tuning the free parameters in the numerical model. In particular, the jet length would be smaller for a smaller amplitude of the initial pulse. We did not expect to fit our numerical data perfectly to the observational findings, but instead our intention was to show a qualitative and to some extent quantitative agreement between the numerical and observational approaches.

Remarkably recently Culhane et al. (2007) have found the first observational evidence of unique EUV polar jet that could not escape from the Sun like the jets previously observed with SXT in the Yohkoh era. These authors conject that this accelerated polar jet plasma is heated up to coronal temperatures with its rise, and then falls back into the lower atmosphere after its cooling. Culhane and coworkers observed jet velocities in the range of $150-360 \mathrm{~km} \mathrm{~s}^{-1}$ and reported that their formation is most probably caused by the magnetic reconnection between emerging bipoles with the large-scale open field lines of the polar coronal hole. Ko et al. (2005) have also found cool and hot plasma components in a limb jet, as well as its falling-back scenario into the lower atmosphere. However, all these previously observed jets were found to be consistent with the impulsive transient heating (e.g., driven by direct reconnection processes), and thereafter cooling and draining along the same path. We also observe the jet motion that moves up and subsequently falls back along the same path into the lower atmosphere. However, the jet consists of the cool plasma maintained at a temperature of $\sim 10^{5} \mathrm{~K}$, which is sensitive to the SDO/AIA $304 \AA$ filter representing the upper chromospheric/TR plasma. However, the jet plasma column is not visible at typical coronal temperatures of $\geq 10^{6} \mathrm{~K}$ in the SDO/AIA 171, $211 \AA$ filters. Therefore, the jet consists of a cool plasma even after reaching at the coronal heights, and is not subject to any heating and cooling events as previously observed by Culhane et al. (2007) and Ko et al. (2005). The primary mechanism for the origin of this jet may be the magnetic reconnection between the emerging bipolar magnetic fields and pre-existing open field lines in the polar coronal hole (Culhane et al. 2007). However, the comparatively slow average rising speed $\left(110 \mathrm{~km} \mathrm{~s}^{-1}\right)$, the steady ballistic motion, the absence of impulsive heating at the base and no gradual enhancement in plasma temperature etc. indicate that the role of magnetic reconnection is an indirect one. The jet is seen to be driven by a large amplitude velocity pulse that may be excited nonlinearly by recurrent magnetic reconnection at the base of the polar coronal hole. This pulse steepens into a slow shock front that moves at a higher temperature wave front. However, the density and typical emissions are low in polar coronal holes. Moreover, the shock propagating into the corona above polar region may leave some traces, although it may be little in a coronal hole because there is no static corona. Therefore, we were unable to observe a high temperature bright front of the jet. Later, the motion of the slow shock, under stable conditions, causes the propagating rarefaction wave, which directs the cool and denser chromospheric/TR plasma upward. This steadily moving plasma is clearly visible in the observations as well as in the simulation. After reaching a height of $\sim 75 \mathrm{Mm}$, the plasma begins to subside. The gravitational free fall causes the falling of jet material.
During the subsiding phase of the jet, another pulse is visible at the base in the numerical simulation that is the reason for the low rate of subsided plasma compared to the observations. However, the realistic solar atmosphere is very complex, and there is a possibility that the huge amount of downfalling material is absorbed in another pulse coming from the lower atmosphere. This is the reason why a pulse-driven single jet was visible above the limb instead of multiple jets at the same place.

In conclusion we suggest that the initial velocity pulse launched below the transition region is able to trigger a hot plasma shock, which is followed by a cool plasma jet. This cold plasma jet roughly resembles many of the features that are found in the SDO/AIA observational data. We report for first time on the observations of a pulse-driven plasma jet in the polar region and provide theoretical explanation of this phenomenon on the basis of numerical simulations. However, more multiwavelength observations should be performed by high-resolution space-borne (e.g., SDO, Hinode, STEREO) and complementary ground-based observations to shed new light on these unique jets that rise and fall back into the solar atmosphere. This will also impose a rigid constraint on the stringent simulations of these jets in the model solar atmosphere.

Acknowledgements. We thank the referee for his/her valuable suggestions that improved the manuscript considerably. We acknowledge the use of the SDO/AIA observations for this study. The data are provided curtesy of NASA/SDO, LMSAL, and the AIA, EVE, and HMI science teams. The FLASH code has been developed by the DOE-supported ASC/Alliance Center for Astrophysical Thermonuclear Flashes at the University of Chicago. A.K.S. acknowledges Shobhna Srivastava for patient encouragement during the work. K.M. thanks Kamil Murawski for his assistance in drawing numerical data.

\section{References}

Alexander, D., \& Fletcher, L. 1999, Sol. Phys., 190, 167

Bohlin, J. D., Vogel, S. N., Purcell, J. D., et al. 1975, ApJ, 197, L133

Brooks, D. H., Kurokawa, H., \& Berger, T. E. 2007, ApJ, 656, 1197

Cirtain, J. W., Golub, L., Lundquist, L., et al. 2007, Science, 318, 1580

Culhane, L., Harra, L. K., Baker, D., et al. 2007, PASJ, 59, 751

De Pontieu, B., Erdélyi, R., \& James, S. P. 2004, Nature, 430, 536

De Pontieu, B., Hansteen, V. H., Rouppe van der Voort, L., van Noort, M., \& Carlsson, M. 2007a, ApJ, 655, 624

De Pontieu, B., McIntosh, S., Hansteen, V. H., et al. 2007b, PASJ, 59, 655

De Pontieu, B., McIntosh, S. W., Carlsson, M., et al. 2011, Science, 331, 55

Filippov, B., Golub, L., \& Koutchmy, S. 2009, Sol. Phys., 254, 259

Georgakilas, A. A., Koutchmy, S., \& Alissandrakis, C. E. 1999, A\&A, 341, 610

Hansteen, V. H., De Pontieu, B., Rouppe van der Voort, L., van Noort, M., \& Carlsson, M. 2006, ApJ, 647, L73

He, J., Marsch, E., Tu, C., \& Tian, H. 2009, ApJ, 705, L217

Innes, D. E., Inhester, B., Axford, W. I., \& Wilhelm, K. 1997, Nature, 386, 811

Kamio, S., Hara, H., Watanabe, T., et al. 2007, PASJ, 59, 757

Kamio, S., Curdt, W., Teriaca, L., Inhester, B., \& Solanki, S. K. 2010, A\&A, $510, \mathrm{~L} 1$

Katsukawa, Y., Berger, T. E., Ichimoto, K., et al. 2007, Science, 318, 1594

Ko, Y.-K., Raymond, J. C., Gibson, S. E., et al. 2005, ApJ, 623, 519

Lee, D., \& Deane, A. E. 2009, J. Comput. Phys., 228, 952

Lemen, J. R., Title, A. M., Akin, D. J., et al. 2011, Sol. Phys., 172

Liu, W., Berger, T. E., Title, A. M., \& Tarbell, T. D. 2009, ApJ, 707, L37

Morton, R., Srivastava, A. K., \& Erdélyi, R. 2011, A\&A, submitted

Murawski, K., \& Zaqarashvili, T. V. 2010, A\&A, 519, A8

Nisticò, G., Bothmer, V., Patsourakos, S., \& Zimbardo, G. 2009, Sol. Phys., 259, 87

Pariat, E., Antiochos, S. K., \& DeVore, C. R. 2009, ApJ, 691, 61

Shibata, K., Ishido, Y., Acton, L. W., et al. 1992, PASJ, 44, L173

Shibata, K., Nakamura, T., Matsumoto, T., et al. 2007, Science, 318, 1591

Shimojo, M., \& Shibata, K. 2000, ApJ, 542, 1100

Vernazza, J. E., Avrett, E. H., \& Loeser, R. 1981, ApJS, 45, 635

Wang, Y.-M., Sheeley, Jr., N. R., Socker, D. G., et al. 1998, ApJ, 508, 899

Yokoyama, T., \& Shibata, K. 1995, Nature, 375, 42

Yokoyama, T., \& Shibata, K. 1996, PASJ, 48, 353 